
ОСВОЕНИЯ БАЗОВЫХ НАВЫКОВ В ЛАПАРОСКОПИЧЕСКОЙ ХИРУРГИИ Ложко П.М. (lpm02091964@yandex.ru),КудлоB.B. (kudloviktor@gmail.com), Киселевский Ю.M. (kiselevski@grsmu.by),ГущаT.C. (gushcha.t@gmail.com) УО «Гродненский государственный медицинский университет», Гродно, Беларусь

Введение. Для освоения лапароскопических навыков в качестве средств обучения используют коробочные тренажеры и виртуальные компьютерные симуляторы разной сложности. Все они имеют свои преимущества и недостатки.

Цель работы. Изучить возможность и эффективность использования созданного на кафедре оригинального видеотренажера для обучения студентов основным лапароскопическим навыкам.

Материал и методы. Исследование выполнено на оригинальном лапароскопическом видеотренажере с привлечением 42 студентов 6 курса. Цифровые данные обработаны с использованием параметрических методов статистического анализа.

Результаты. Выполнение упражнений после двукратных тренировок дало достоверное улучшение, в частности, по критерию скорости (времени). Субъективно большинство испытуемых стали действовать более четко и уверенно, уменьшилось количество лишних движений и ошибок.

Bыводы. Лапароскопический тренажер эффективен для обучения базовым эндовидеохирургическим манипуляциям и может использоваться в учебном процессе студентами старших курсов на первом этапе их отработки, предшествующем занятиям на тренажере виртуальной реальности.

Ключевые слова: видеотренажер, базовые навыки, лапароскопическая техника, обучение.

\section{введение}

Одна из важнейших тенденций развития хирургии в последние десятилетия - стремление к уменьшению травматичности оперативного доступа при выполнении оперативного вмешательства. Все свои преимущества в решении этой проблемы демонстрирует эндовидеохирургия - метод оперативного лечения заболеваний, когда радикальные вмешательства выполняют без широкого рассечения покровов, через точечные проколы тканей с выведением изображения на экран монитора. Данная методика выполнения операций имеет свои особенности в силу ряда причин (двухмерное изображение объекта, отсутствие ощущения «глубины», наличие «эффекта качелей» и др.), требует использования специального оборудования и инструментария, с которым надо уметь работать. За более чем тридцатилетний период с момента выполнения первой лапароскопической холецистэктомии в клинике (Ф. Муре, 1987 г.) подготовка хирургов в данной области прошла ряд этапов. До настоящего времени актуален метод наставничества, когда начинающий хирург учится у опытного хирурга, вначале наблюдая за ходом операции, затем ассистируя на видеокамере, вспомогательных инструментах. По мере приобретения навыков ему доверяют выполнение основных манипуляций (рассечение тканей, гемостаз и др.) и самостоятельное выполнение операции под контролем наставника.

Развитие эндовидеохирургии и стремительное внедрение лапароскопических и малоинвазивных операций в клиническую практику стимулировало создание в качестве средств обучения симуляционных тренажеров и виртуальных компьютерных программ. Наиболее часто на практике применяются «коробочные» тренажеры и компьютерные симуляторы [1]. Первые из них представляют собой прямоугольную коробку с фиксированными отверстиями, через которые вводятся троакары или непосредственно эндохирургические инструменты. Данные тренажеры помогают обучающемуся адаптироваться к работе в условиях двухмерного изображения, транслируемого на монитор, научиться работать с лапароскопическими инструментами в закрытом пространстве, координировать движение рук, и предназначены для отработки базовых лапароскопических навыков, включающих пять упражнений по системе FLS (Fundamentals of Laparoscopic Skills) [2]. Данные симуляционные устройства относительно недороги и просты в эксплуатации. К недостаткам этого вида тренажеров следует отнести необходимость присутствия преподавателя для контроля правильности выполнения манипуляций и внесения корректирующих действий во избежание закрепления ошибок при освоении навыка. Последующая генерация лапароскопических тренажеров имеет эндоскопическую стойку и муляж, имитирующий брюшную полость с ее содержимым. Данный вид дает возможность выполнить лапароскопическую операцию, начиная с введения троакаров и заканчивая ушиванием лапароскопических отверстий.

Наиболее сложным классом тренажеров являются роботизированные симуляторы виртуальной реальности, которые основаны на компьютерных программах и имитируют виртуальные модели полостей человеческого тела с их содержимым, а также способны моделировать картину при различной хирургической патологии. Выполнение отдельных манипуляций и оперативных приемов на подобных тренажерах не требует присутствия преподавателя, так как симулятор в автономном режиме контролирует работу: дает методические подсказки, указыва- 
ет на ошибки и оценивает выполненную работу. Высокая эффективность виртуального тренинга в освоении базовых практических навыков эндовидеохирургических вмешательств доказана исследованиями, на основании чего его считают основным средством обучения [3]. Однако что касается тактильной чувствительности, необходимой при оперативных вмешательствах, специалисты в области эндохирургии пришли к единому мнению о том, что лапароскопический «коробочный» тренажер имеет преимущество перед системами симуляции виртуальной реальности при отработке навыков наложения лапароскопических швов, так как они недостаточно реалистичны и не обеспечивают тактильную чувствительность в полной мере [4]. Существенным недостатком, сдерживающим широкое внедрение в практику обучения в Республике Беларусь подобных виртуальных симуляционных технологий, является их высокая стоимость. Кроме того, симуляторы виртуальной реальности требуют весьма бережного обращения при работе с ними. Показано, что комбинация «коробочных» тренажёров и виртуальных симуляторов приводит к наилучшему освоению навыков, нежели использование этих методов по отдельности, поэтому использование дорогостоящих виртуальных симуляторов должно проводиться параллельно с более простыми тренажёрами [5, 6]. Психомоторные навыки улучшаются после тренинга как на видеотренажере, так и на тренажере виртуальной реальности. Практическое обучение на обоих видах тренажеров может повышать оперативное мастерство [7].

В настоящее время для практической подготовки эндохирургов разработана и используется система, включающая последовательную цепочку этапов обучения: изучение теории видеохирургии; освоение базовых навыков на симуляторах; освоение базовых навыков на эндоскопических симуляторах (видеотренажёрах); операции на симуляторах высокого уровня реалистичности; ассистенция (на видеокамере, на вспомогательных инструментах); самостоятельное выполнение оперативного вмешательства под контролем преподавателя на животных в экспериментальной операционной; клиническая работа в операционной [8].

Цель исследования: изучить возможность и эффективность использования созданного на кафедре оригинального видеотренажера для обучения студентов основным лапароскопическим навыкам.

\section{Материал и методы}

Исследование выполнено на оригинальном лапароскопическом видеотренажере (удостоверение на рационализаторское предложение № 1661 от 12.02.2014 г.), включающем фантом торса на основании, компьютер, монитор, набор лапароскопических инструментов, сменные модули для манипуляций, сделанные сотрудниками кафедры с привлечением 42 студентов-субординаторов, обучающихся на 6 курсе лечебного факультета.
Основным компонентом тренажера является фантом торса на основании, состоящий (в соответствии с рисунком 1) из двух частей: плоского основания и торса из непрозрачного материала со срезанной во фронтальной плоскости задней частью.

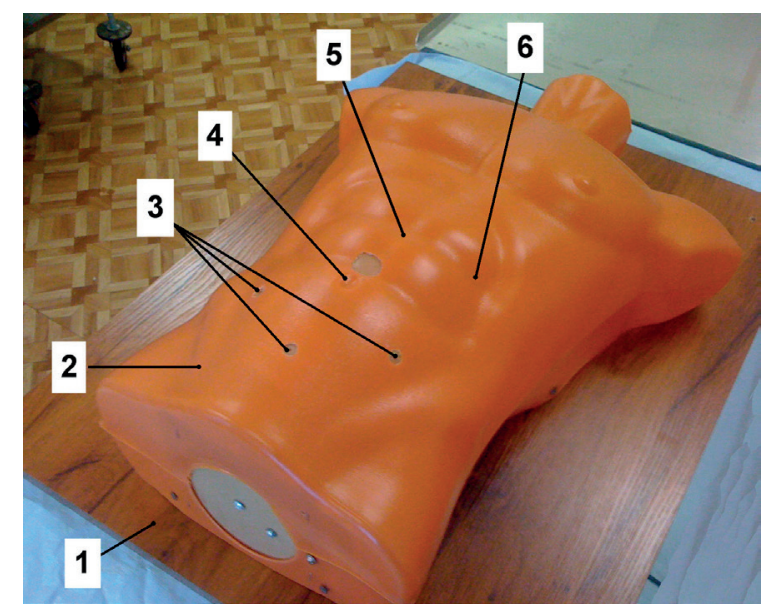

1 - плоское основание; 2 - торс; 3 -отверстия для лапароскопических инструментов; 4 - пупочное кольияо; 5 - белая линия живота; 6 - прямая мышича живота

Рисунок 1. - Фантом торса на основании

На передней стенке торс снабжен отверстиями для проведения лапароскопических инструментов или установки троакаров, соответствующими разным лапароскопическим доступам в соответствии с анатомическими ориентирами: пупочным кольцом, белой линией живота, прямыми мышцами живота. Объем имитатора брюшной полости соответствует объему пневмоперитонеума.

Для удобства в работе торс подвижно прикрепляется к угловым фиксаторам, закрепленным на основании с помощью шурупов (рис. 2). С внутренней стороны к стенке торса фиксирован источник искусственного света - лампочка, подвижно на тубусе лапароскопа крепится широкоформатная web-камера с возможностью движений во всех направлениях. От лампочки и web-камеры тянутся провода, выходящие наружу через отверстие в основании для подключения к источнику переменного тока и компьютеру.

Основание фантома торса с внутренней стороны служит рабочей поверхностью. Для размещения на рабочей поверхности изделий для манипуляций фантом торса легко снимается с угловых фиксаторов.

Лапароскопический тренажер используют следующим образом. В полость фантома торса укладываются сменные модули для манипуляций, крючки, петли для отработки эндоскопического узла, искусственные или анатомические материалы для отработки навыков эндоскопического шва, рассечения тканей и т. д. После включения компьютера и источника искусственного света в соответствии с выполняемой манипуляцией выбирается доступ на передней брюшной стенке фантома торса, после чего инструменты вводятся в соответствующие отверстия. Обу- 


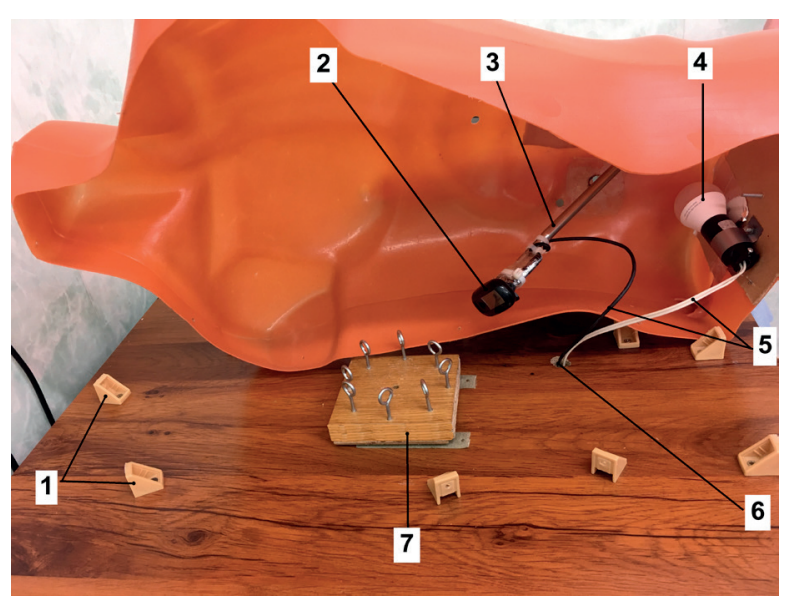

1 -угловые фиксаторы; 2 - жеb-камера; 3 - тубус лапароскопа; 4-лампочка; 5-провода; 6-отверстие в плоском основании; 7 -сменный модуль

\section{Рисунок 2. - Фантом торса на основании (вид изнутри)}

чаемый под контролем преподавателя, ориентируясь по изображению на экране монитора, осуществляет необходимые манипуляции лапароскопическими инструментами.

Сменные модули для манипуляций представляют собой простые в изготовлении изделия, легко и быстро фиксируемые на рабочей поверхности тренажера застежкой-липучкой, позволяющие осуществлять следующие манипуляции: сбор муляжей конкрементов в контейнер, перемещение объектов с перехватом другим зажимом на весу, продевание нити через кольца с использованием двух зажимов, вязание интракорпорального узла, рассечение ткани ножницами (рис. 3).

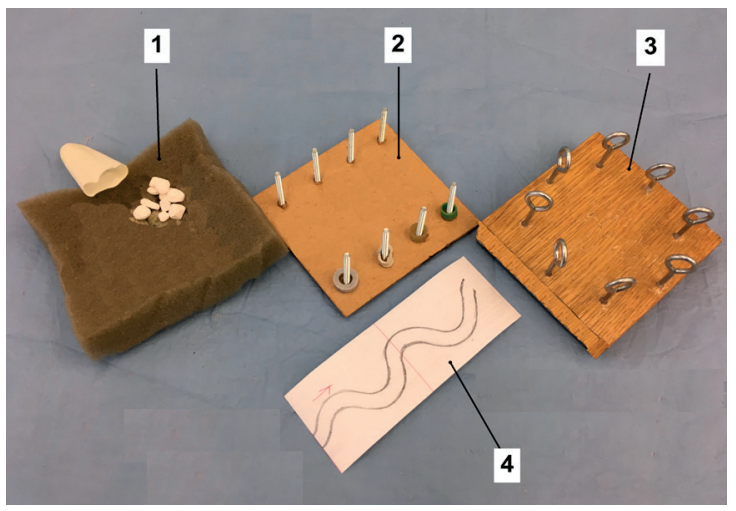

1 -модуль для сбора муляжей конкрементов в контейнер; 2 -модуль для перемещчения объектов; 3 -модуль для продевания нити через кольија; 4 -модуль для рассечения ткани ножницами

\section{Рисунок 3. - Сменные модули для манипуляций}

Этапность исследования. На первом занятии цикла студенты группы после вводного инструктажа о работе на лапароскопическом тренажере и демонстрации преподавателем каждой манипуляции самостоятельно, без предварительной тренировки, выполняли требуемые действия. При этом один участник контролировал поло- жение видеокамеры, а второй - инструментами выполнял манипуляцию. Ограничения по времени не было. Преподаватель контролировал правильность, уверенность, четкость выполняемых действий, указывал на ошибки, делал замечания корректирующего характера и фиксировал время, затраченное на выполнение манипуляции. На втором и третьем занятиях все студенты под контролем преподавателя тренировались в совершенствовании ранее выполненных манипуляций с учетом полученных замечаний. На тренировку по освоению отдельного навыка приходилось около 5 минут на одного участника за занятие. На четвертом занятии каждый студент, участник исследования, демонстрировал уровень освоения предложенных лапароскопических манипуляций с контролем времени, правильности и уверенности выполненной работы и фиксацией допущенных ошибок.

Полученные цифровые данные обрабатывались в программе Microsoft Excel 2013 с исполь-

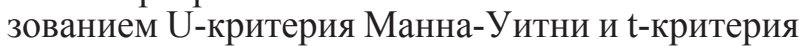
Стьюдента. Разница между сравниваемыми показателями считалась статистически достоверной при $\mathrm{p}<0,05$.

\section{Результаты и обсуждение}

Время выполнения упражнений фиксировалось в секундах и представлено в таблице.

Tаблица. - Цифровая оценка скорости выполнения тренировочных упражнений

\begin{tabular}{|c|c|c|}
\hline $\begin{array}{c}\text { Оцениваемое } \\
\text { упражнение }\end{array}$ & \multicolumn{2}{|c|}{ Время выполнения, сек } \\
\hline \multirow{2}{*}{$\begin{array}{l}\text { 1. Сбор муляжей } \\
\text { конкрементов }\end{array}$} & до тренировки & $112,0 \pm 84,1$ \\
\hline & $\begin{array}{c}\text { после } \\
\text { тренировки }\end{array}$ & $66,3 \pm 22,3 *$ \\
\hline \multirow{2}{*}{$\begin{array}{l}\text { 2. Проведение нити } \\
\text { через кольца }\end{array}$} & до тренировки & $222,7 \pm 81,0$ \\
\hline & $\begin{array}{c}\text { после } \\
\text { тренировки }\end{array}$ & $150,4 \pm 47,7^{*}$ \\
\hline \multirow{2}{*}{$\begin{array}{l}\text { 3. Перемещение } \\
\text { объектов }\end{array}$} & до тренировки & $183,2 \pm 90,9$ \\
\hline & $\begin{array}{c}\text { после } \\
\text { тренировки }\end{array}$ & $108,9 \pm 45,9 *$ \\
\hline \multirow{2}{*}{$\begin{array}{l}\text { 4. Интракорпоральный } \\
\text { узел }\end{array}$} & до тренировки & $259,0 \pm 134,4$ \\
\hline & $\begin{array}{c}\text { после } \\
\text { тренировки }\end{array}$ & $157,8 \pm 78,5^{*}$ \\
\hline \multirow{2}{*}{$\begin{array}{l}\text { 5. Рассечение } \\
\text { ножницами }\end{array}$} & до тренировки & $272,8 \pm 163,4$ \\
\hline & $\begin{array}{c}\text { после } \\
\text { тренировки }\end{array}$ & $179,1 \pm 111,0^{*}$ \\
\hline
\end{tabular}

Примечание - * статистически достоверное снижение оцениваемого параметра

При сборе муляжей конкрементов в контейнер после тренировок снижение среднего времени выполнения манипуляции составило 1,7 раза $(\mathrm{p}<0,05)$. Кроме того, у 95,2\% студентов продолжительность проведения упражнения сократилась по сравнению с его первоначальным выполнением. При проведении нити через кольца с использованием двух зажимов средняя продолжительность задания после тренировок сократилась в 1,5 раза $(\mathrm{p}<0,05), 83,3 \%$ участни- 
ков улучшили свой результат. Время перемещения объектов с перехватом зажимом на весу при контрольной оценке уменьшилось в 1,7 раза $(p<0,05)$. Снижение относительной разницы времени выполнения манипуляции отмечено у 90,5\% студентов. Продолжительность формирования интракорпорального узла после тренировок сократилась у $88,1 \%$ участников, а по сравнению с первичным выполнением снизилась в 1,6 раза $(\mathrm{p}<0,05)$. Рассечение муляжа ножницами после двукратной тренировки потребовало в 1,5 раза $(\mathrm{p}<0,05)$ меньше времени, чем при первоначальном выполнении, а большая часть студентов-субординаторов $(80,7 \%)$ улучшили результат. Таким образом, при выполнении манипуляций после двукратных непродолжительных тренировок очевидно достоверное улучшение, в частности по критерию скорости (времени). Следует отметить, что некоторые участники исследования ухудшили результаты выполнения манипуляции после тренировки. При сборе муляжей конкрементов и перемещении объектов это сделали около 5\% студентов, при вязании узлов $-12 \%$, при рассечении тканей - около $19 \%$, а при проведении нити через кольца $-16 \%$.

Кроме объективных критериев оценки учитывались и субъективные. При контрольном выполнении тренировочных упражнений большинство испытуемых действовали более четко и уверенно, уменьшилось количество лишних движений и ошибок. В частности, при сборе муляжей конкрементов в контейнер первоначально было допущено 16 ошибок (падение объекта на рабочую поверхность фантома торса), а при контрольной оценке все студенты успешно справились с заданием. При перемещении объектов с перехватом зажимом на весу падение колец произошло в 30 случаях до тренировки и в 7 случаях после тренировки. Из данных семи случаев четыре - это повторные ошибки одних и тех же испытуемых. При рассечении ткани ножницами

\section{Литература}

1. Randomized clinical trial of virtual reality simulation for laparoscopic skills training / T. R. Grantcharov [et al.] // Br. J. Surg. - 2004. - Vol. 91, № 2. - P. 146-150.

2. Development of a model for training and evaluation of laparoscopic skills / A. M. Derossis [et al.] // Am. J. Surg. 1998. - Vol. 175, № 6. - P. 482-487.

3. Федоров, А. В. Отработка базовых эндохирургических навыков на виртуальных тренажерах. Обзор литературы / А. В. Федоров, М. Д. Горшков // Виртуальные технологии в медицине. - 2009. - Т. 2, № 2. - С. 16-28.

4. The importance of haptic feedback in laparoscopic suturing training and the additive value of virtual reality simulation / S. M. Botden [et al.] // Surg. Endosc. - 2007. - Vol. 194, № 22. - P. 1214-1222.

5. Оптимизация обучения лапароскопической хирургии в условиях центра непрерывного профессионального образования / А. А. Свистунов [и др.] // Виртуальные технологии в медицине. - 2012. - Т. 7, № 1. - С. 27-34.

6. Madan, A. K. Prospective randomized controlled trial of laparoscopic skills acquisition / A. K. Madan, C. T. Frantzides // Surg. Endosc. - 2007. - Vol. 193,

№ 21. - P. 209-213.
8 студентов допустили ошибку (выход линии разреза за допустимые границы), из них двое повторили ее и после тренировки. Вышеописанные погрешности в выполнении упражнений, а также увеличение затраченного на них времени некоторыми студентами можно объяснить несколькими причинами: неравномерное распределение тренировочного времени в учебной группе и его недостаточность, эмоциональное волнение во время контроля, индивидуальные сложности в перестройке системы «зрительный анализатор-монитор-руки» с трехмерного на двухмерный уровень.

Лапароскопический тренажер активно используется в учебное время при изучении студентами 6 курса дисциплин по выбору, а также после занятий. На нем осуществляют тренировку студенты 4-6 курсов в рамках работы студенческого научного кружка кафедры, при подготовке к эндохирургическому конкурсу университетской и республиканской предметных олимпиад по оперативной хирургии и топографической анатомии. Именно благодаря тренировке на указанном тренажере студенты нашего университета, по их мнению, заняли 1 место в лапароскопическом конкурсе на Республиканской межвузовской олимпиаде по акушерству и гинекологии в 2016 г.

\section{Выводы}

1. Лапароскопический тренажер показал высокую эффективность его использования для обучения базовым эндовидеохирургическим манипуляциям. Подавляющее большинство испытуемых достоверно улучшили время их выполнения.

2. Данный тренажер может использоваться в учебном процессе студентами старших курсов на первом этапе отработки лапароскопических манипуляций, предшествующем занятиям на тренажере виртуальной реальности.

7. Comparison of video trainer and virtual reality training systems on acquisition of laparoscopic skills / E. S. Hamilton [et al.] // Surg. Endosc. - 2002. - Vol. 16, № 3. - P. 406-411.

8. Горшков, М. Д. Принципы построения обучающего симуляционного курса по основам лапароскопической хирургии / М. Д. Горшков, Ю. И. Логвинов // Виртуальные технологии в медицине. - 2015. - Т. 13, № 1 - С. 16-23.

\section{References}

1. Grantcharov TP, Kristiansen VB, Bendix J, Bardram L, Rosenberg J, Funch Jensen P. Randomized clinical trial of virtual reality simulation for laparoscopic skills training. $\mathrm{Br}$. $J$. Surg. 2004;91(2):146-150.

2. Derossis AM, Fried GM, Abrahamowicz M, Sigman HH, Barkun JS,

Meakins JL. Development of a model for training and evaluation of laparoscopic skills. Am. J. Surg. 1998;175(6):482487.

3. Fedorov AV, Gorshkov MD. Otrabotka bazovyh jendohirurgicheskih navykov na virtual'nyh trenazherah. Obzor literatury [Development of basic endosurgical skills in virtual simulators. Literature review]. Virtual'nye tehnologii v medicine [Virtual technologies in medicine]. 2009;2(2):16-28. (Russian). 
4. Botden SM, Torab F, Buzink SN, Jakimowicz JJ. The importance of haptic feedback in laparoscopic suturing training and the additive value of virtual reality simulation. Surg. Endosc. 2007;194(22):1214-1222.

5. Svistunov AA, Kossovich MA, Vasilev MV, Shubina LB, Gribkov DM. Optimizacija obuchenija laparoskopicheskoj hirurgii $\mathrm{v}$ uslovijah centra nepreryvnogo professional'nogo obrazovanija [Optimization of the training of laparoscopic surgery in the context of the center for continuing vocational education]. Virtualnye tehnologii $v$ medicine [Virtual technologies in medicine]. 2012;7(1):27-34. (Russian).

6. Madan AK, Frantzides CT. Prospective randomized controlled trial of laparoscopic skills acquisition. Surg. Endosc.
2007;193(21):209-213.

7. Hamilton EC, Scott DJ, Fleming JB, Rege RV, Laycock R, Bergen PC, Tesfay ST, Jones DB. Comparison of video trainer and virtual reality training systems on acquisition of laparoscopic skills. Surg. Endosc. 2002;16(3):406-11.

8. Gorshkov MD, Logvinov JuI. Principy postroenija obuchajushhego simuljacionnogo kursa po osnovam laparoskopicheskoj hirurgii [Principles of constructing a training simulation course on the basics of laparoscopic surgery]. Virtualnye tehnologii $v$ medicine [Virtual technologies in medicine]. 2015;13(1):16-23. (Russian).

\title{
THE EXPERIENCE OF USING OF THE ORIGINAL VIDEO SIMULATOR FOR THE DEVELOPMENT OF BASIC SKILLS IN LAPAROSCOPIC SURGERY
}

\author{
Lozhko P. M., Kudlo V. V., Kiselevskiy Yu. M., Gushcha T. S.
}

Educational Institution "Grodno State Medical University", Grodno, Belarus

\begin{abstract}
Introduction. For development of laparoscopic skills, box training simulators and virtual computer simulators of different complexity are used as training tools. All of them have advantages and disadvantages.

Objective. To study the possibility and efficiency of department-created original video simulator for providing basic laparoscopic skills to students.

Material and methods. The study was performed on the original laparoscopic video simulator with the participation of 42 students of the 6th year of studying. The digital data processed using the parametric methods of statistical analysis.

Results. Exercises after two training gave a significant improvement on the criterion of speed (time). Subjectively, most of participants began to perform them more clearly and confidently, the number of unnecessary movements and mistakes decreased.

Conclusions. The laparoscopic simulator is effective for training of basic endovideosurgical manipulations and can be used in educational process by the senior students at a preliminary stage before classes on simulator of virtual reality.
\end{abstract}

Keywords: video simulator, basic skills, laparoscopic technique, training. 